\title{
Boron abundances in the Galactic disk
}

\author{
Katia Cunha ${ }^{1}$ \\ ${ }^{1} \mathrm{NOAO}$ \\ 950 N. Cherry Ave, Tucson, Arizona \\ email: kcunha@noao.edu
}

\begin{abstract}
When compared to lithium and beryllium, the absence of boron lines in the optical results in a relatively small data set of boron abundances measured in Galactic stars to date. In this paper we discuss boron abundances published in the literature and focus on the evolution of boron in the Galaxy as measured from pristine boron abundances in cool stars as well as early-type stars in the Galactic disk. The trend of B with Fe obtained from cool F-G dwarfs in the disk is found to have a slope of $0.87 \pm 0.08$ (in a log-log plot). This slope is similar to the slope of $\mathrm{B}$ with Fe found for the metal poor halo stars and there seems to be a smooth connection between the halo and disk in the chemical evolution of boron. The disk trend of boron with oxygen has a steeper slope of 1.5. This slope suggests an intermediate behavior between primary and secondary production of boron with respect to oxygen. The slope derived for oxygen is consistent with the slope obtained for Fe provided that $[\mathrm{O} / \mathrm{Fe}]$ increases as $[\mathrm{Fe} / \mathrm{H}]$ decreases, as observed in the disk.
\end{abstract}

Keywords. stars: abundances - Galaxy: disk - ultraviolet: stars

\section{Introduction}

The light element boron is one of the few elements whose production is not dominated by nucleosynthesis in stars, nor by nucleosynthesis occurring in the Big Bang. In fact, it has been known now for almost 4 decades that Galactic Cosmic Rays are related to the formation of the light elements and, in particular, of boron (Reeves, Fowler \& Hoyle 1970). The connection between boron production and cosmic rays spallation reactions, which involve $\mathrm{C}, \mathrm{N}, \mathrm{O}$ atoms, as well as protons and $\alpha$ particles, makes boron an interesting element whose abundance evolution in the Galaxy probes the history of cosmic rays in the galactic environment. In addition, boron is also proposed to be produced by neutrino nucleosynthesis occurring in core collapse of Supernovae Type II (Woosley et al. 1990).

Unveiling the underlying behavior of boron with metallicity (iron and oxygen abundances) is crucial in order to constrain models for boron production. One of the challenges in trying to pin down the behavior of boron with metallicity, however, comes first from the fact that boron is fragile and easily destroyed in stellar interiors (although sturdier than $\mathrm{Li}$ and $\mathrm{Be}$ ) and, in addition, from the difficulty in obtaining boron observations. In this paper we discuss stellar boron abundance results mainly for disk stars which have been published in the literature. Unfortunately, no new boron observations were available in recent years due to the failure of STIS on board HST.

\section{Boron transitions and abundance determinations}

Boron abundance results are still sparse as boron abundances can only be measured from transitions which fall mainly in the ultraviolet. Boron abundance indicators in different temperature regimes are from three ionization stages: neutral boron in cool

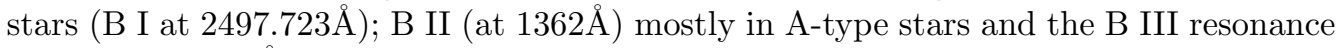
doublet (at $2060 \AA$ ) in B-type stars. 


\subsection{Boron in cool stars and the Sun}

Boron was measured in the Sun by Kohl, Parkinson \& Withbroe (1977). One of the pioneering studies of boron abundances in stars was by Boesgaard \& Heacox (1978). A few studies appeared more than a decade later from observations obtained with the Hubble Space Telescope (Duncan, Lambert, \& Lemke 1992; Duncan et al. 1997; Primas et al. 1999). The sample analyzed in Duncan et al. (1997) was mostly for halo stars. Their results were particularly important as they found that boron abundances scaled linearly (in log-log space) with the abundance of metals, in contrast with predictions from the standard models of cosmic ray production, which proposed a secondary behavior for boron with metallicity. These predictions from cosmic ray models had remained unchallenged for $\sim 20$ years.

Following studies focused on samples of stars with disk metallicities and, by the nature of their sample, these probed the behavior of boron in the most metal rich stars in the Galaxy (Boesgaard et al. 1998; Cunha \& Smith 1999; Cunha et al. 2000; Boesgaard et al. 2004; Boesgaard et al. 2005). It is important to acknowledge, however, that the line list in the spectral region of the B I transition is a major challenge for the analysis of boron in solar-like stars as the spectral region to be synthesized is covered with strong blending lines for which, in many instances, there is no atomic data available. Note, however, that this does not represent a severe problem for the analysis of halo stars as the metal

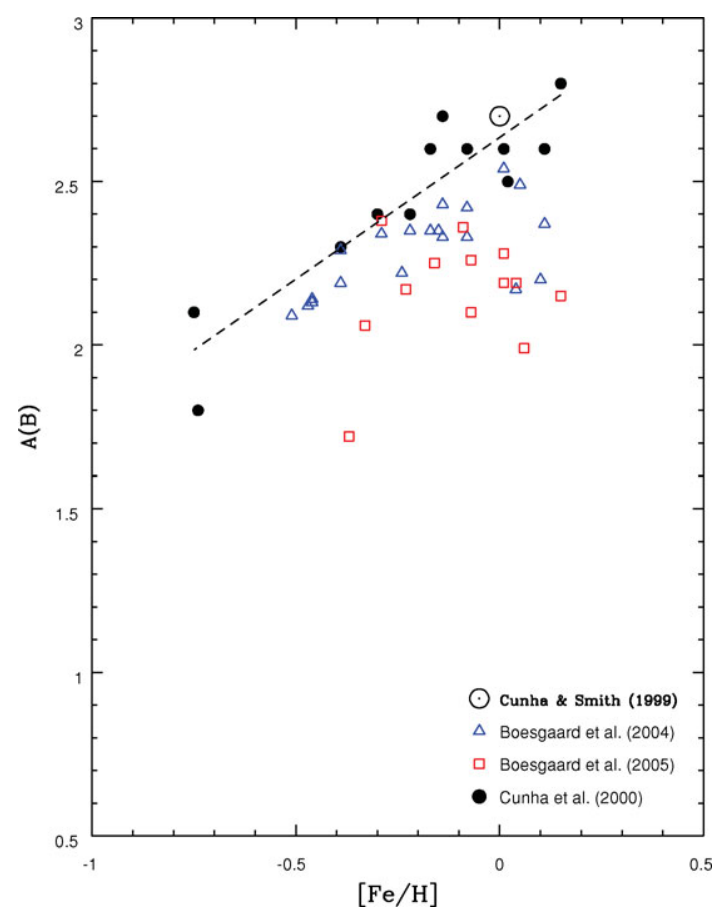

Figure 1. Boron results for the available samples of F-G disk stars. The solar abundance is also plotted. The targets in Boesgaard et al. (2004) and Cunha et al. (2000) are beryllium undepleted, while the stars analyzed in Boesgaard et al. (2005) are mixed showing a degree of Be depletion. For the Be undepleted stars it is clear that there is a systematic abundance offset between the studies of Cunha et al. (2000) and Boesgaard et al. (2004) which can be attributed to the differences in the line lists adopted in the analyses. The slope representing the trend of boron with iron for the disk is $0.87 \pm 0.08$ (dashed line); this defines the upper envelope (undepleted) of the distribution. 
lines are vanishingly weak. For solar metallicity stars, different studies in the literature constructed and adopted different line lists which resulted in systematic differences in the derived boron abundances. In the following we briefly present some of the boron results in these studies.

Cunha et al. (2000) analyzed dwarf stars with $[\mathrm{Fe} / \mathrm{H}]>-1.0\left(\mathrm{~T}_{\text {eff }}\right.$ 's between 5650 $6700 \mathrm{~K})$ from HST archival data. One important aspect of that study in comparison with Boesgaard et al. $(1998 ; 2004)$ is that the line list adopted in the calculation of model spectra in Cunha et al. (2000) was empirically adjusted in order to fit the Sun. In using the Sun as a benchmark in the study of solar-type stars, Cunha \& Smith (1999) re-visited the analysis of boron in the solar photosphere. In particular, significant effort was put in that study into evaluating and updating the opacities which are important in the ultraviolet and which affect the derived boron abundances. This resulted in the revision upwards of the boron abundance in the solar photosphere, which was found to be in good agreement with the boron meteoritic value of $\mathrm{A}(\mathrm{B})=2.79 \pm 0.04$ ( see Lodders, Palme \& Gail 2009). The agreement between the boron abundances in the solar photosphere and meteorites (implying an absence of boron depletion in the Sun) is an important result as it reconciles with the most recent assessment of the beryllium abundance in the solar photosphere by Asplund et al. (2009), indicating no beryllium depletion in the Sun. If Be is indeed not depleted in the solar photosphere, it follows that boron, which is less fragile than Be, cannot be depleted in the solar photosphere.

Boron abundances for disk dwarfs with effective temperatures close to solar are shown in Figure 1. Non-LTE corrections for the B I transition at $2497 \AA$ for this temperature range at solar metallicity are deemed to be small (Kiselman \& Carlson 1996). In order to have all stars and the Sun on a consistent scale, all disk stars in Cunha et al. (2000) were analyzed homogeneously. The study by Boesgaard et al. (2004) used a different line list which was not fine tuned in order to fit the solar spectrum. It can be seen that the results from Cunha et al. (2000) and Boesgaard et al. (2004), all for targets with undepleted beryllium, have a small systematic offset. It is clear also that the targets analyzed Boesgaard et al. (2005) have significantly lower boron abundances, but this is expected as the target stars were selected in that study to be beryllium depleted in order to further study mixing.

\subsection{Boron in early-type stars}

Of the light element trio, boron is the only element whose abundance can be measured in early-type stars. One problem in using early-type stars to define the boron Galactic trend, however, is the varying amounts of boron depletion in OB-type stars, as depletion of boron is proportional to stellar mass, age and rotational velocity. In addition, unlike the case of observations of $\mathrm{Li}$ and $\mathrm{Be}$ in cool stars, there is not a sensitive monitor of depletion in early-type stars. Boron is burnt at temperatures which are lower than those at which the $\mathrm{CN}$ cycle takes place and is much more sensitive to mixing than nitrogen.

Some studies in the literature have derived boron abundances from HST observations obtained with the GHRS and STIS spectrographs in relatively small samples of earlytype stars (Cunha et al. 1997; Venn et al. 2002; Mendel et al. 2006). Most of these boron abundances, however, were found to be somewhat mixed and therefore not representative of the chemical composition of the gas which formed these young stars. The larger sample analyzed by Proffitt \& Quigley (2001) from IUE archival observations of the B III resonance line at $2066 \AA$, although not having the same spectral quality as HST data, found some stars to be boron undepleted which helped define the disk trend. 


\section{Boron abundance trends in the disk}

The evolution of boron with oxygen for metallicities covering the range spanned by the disk is shown in Figure 2. The blue filled circles represent the FG-dwarfs analyzed in Cunha et al. (2000) with the errorbars indicating the estimated abundance uncertainties. Boron results for early-type stars from Proffitt et al. (2001; filled red triangles) and Mendel et al. (2006; filled red squares) are also shown. Most of the B stars shown have roughly undepleted boron and on average follow the disk trend delineated by the cool stars. The general agreement between the results in cool and hot stars is pleasing given that the physical conditions in their stellar atmospheres are quite distinct. The behavior of boron with oxygen can be represented by a linear relation (in the log-log plot) with a slope $\sim 1.5$, which can suggest an intermediate behavior between primary and secondary production for boron with respect to oxygen.

In Figure 3 we plot boron abundances versus $[\mathrm{Fe} / \mathrm{H}]$ spanning the metallicity range from the halo to the disk. If we adopt the boron abundances for the Be undepleted stars from Cunha et al. (2000; filled circles) as representative of the disk value, there seems to be a smooth transition between the halo and disk which follows a slope of $\sim 0.9$. This slope for the disk + halo is closer to a primary rather than a secondary behavior for boron production. In addition, it is important to note that the slope derived for oxygen (from Figure 2) is consistent with the one obtained for Fe provided that $[\mathrm{O} / \mathrm{Fe}]$ increases as $[\mathrm{Fe} / \mathrm{H}]$ decreases, as observed in the disk.

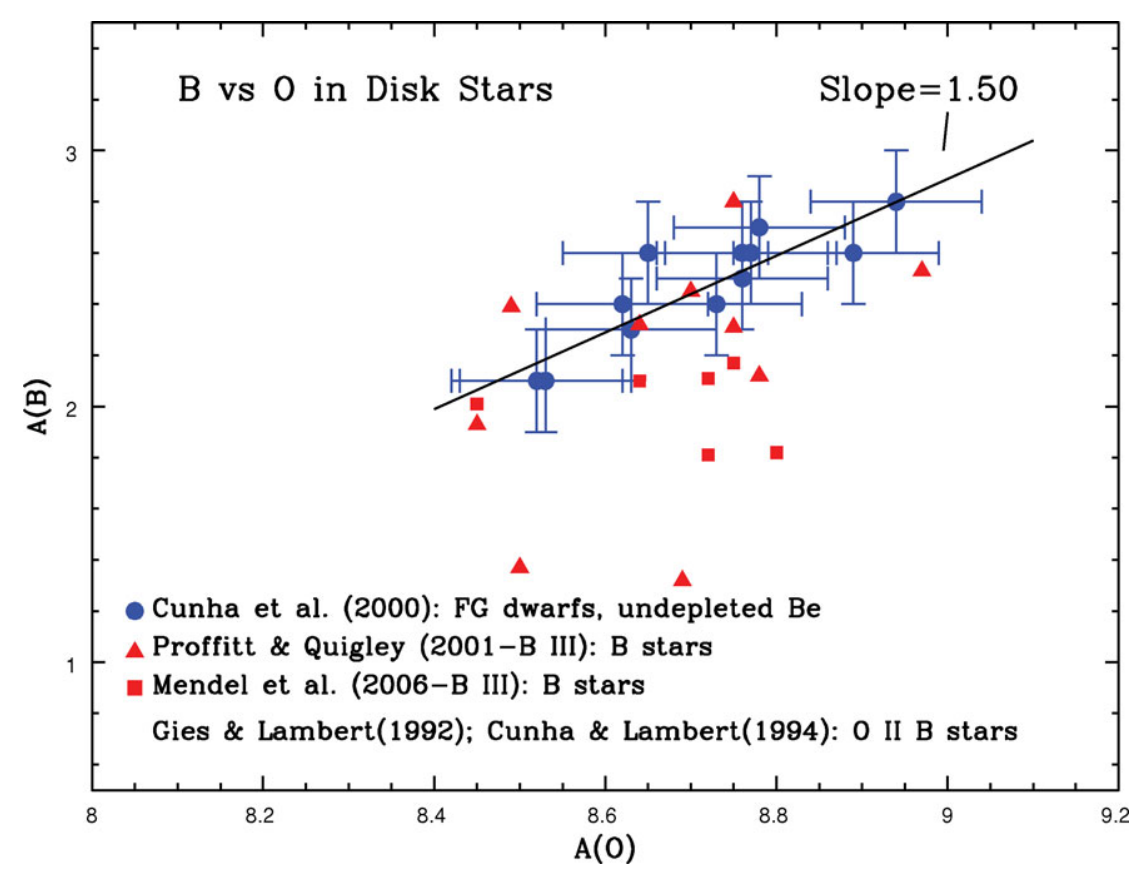

Figure 2. The evolution of boron and oxygen abundances in the Galactic disk. Boron results for cool disk FG-type dwarfs are taken from Cunha et al. (2000); these targets have undepleted Be abundances which indicate that their boron content is not mixed and representative of their natal clouds. The evolution of boron and oxygen from this dataset can be represented by linear relation with slope $\sim 1.5$. Boron results for early-type stars from Proffitt \& Quigley (2001) and Mendel et al. (2006) are also shown. The lower boron abundances in some of the B-type stars are due to internal mixing and astration. 


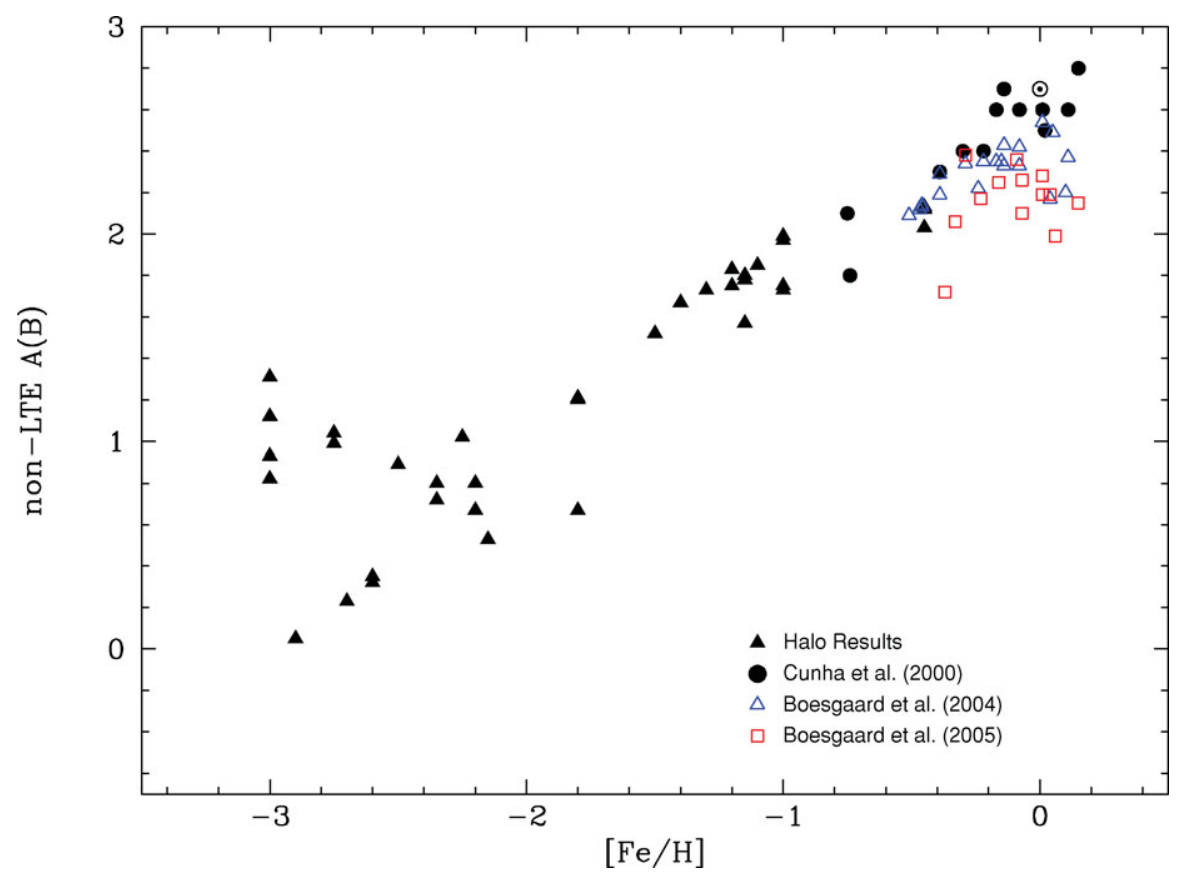

Figure 3. The behavior of boron with metallicity for the Galactic disk in comparison with the trend obtained for the more metal poor stars in the halo. If the abundance results in Cunha et al. (2000) are adopted as representative of the disk (filled circles), there seems to be a smooth transition between the disk and halo. The halo star abundances are taken from Duncan et al. (1997) and García López et al. (1998).

\section{How do we move forward?}

Probing the behavior of boron with metallicity is crucial for understanding boron production in the Galaxy and the relatively small number of stars analyzed to date could usefully be increased. It is good news that the STIS spectrograph has been recently fixed in a very successful NASA servicing mission (SM4) and that it can now be ready for more boron observations in the UV. In addition, the new UV Cosmic Origins Spectrograph (COS) was deployed on HST during SM4. We also need improvements in the abundance analysis and in particular improvements in the line lists to model the boron region. Full non-LTE treatment is needed, including non-LTE analysis of transitions of all elements contributing to the boron blend; and a complete hydrodynamic 3-D modelling of the stellar atmospheres is also something to look forward to in particular for the cool stars.

\section{References}

Asplund, M., Grevesse, N., Sauval, A. J., \& Scott, P. 2009, ARAA, 47, 481

Boesgaard, A. M. \& Heacox, W. D. 1978, ApJ, 226, 888

Boesgaard, A. M., Deliyannis, C. P., Stephens, A., \& Lambert, D. L. 1998, ApJ, 492, 727

Boesgaard, A. M., McGrath, E. J., Lambert, D. L., \& Cunha, K. 2004, ApJ, 606, 306

Boesgaard, A. M., Deliyannis, C. P., \& Steinhauer, A. 2005, ApJ, 621, 991

Cunha, K., Lambert, D. L, Lemke, M., Gies, D. R., \& Lewis, C. R. 1997, ApJ, 478, 211

Cunha, K. \& Smith, V. V. 1999, ApJ, 512, 1006

Cunha, K., Smith, V. V., Boesgaard, A. M., \& Lambert, D. L. 2000, ApJ, 530, 939

Duncan, D. K., Lambert, D. L., \& Lemke, M. 1992, ApJ, 401, 584 
Duncan, D. K., Primas, F., Rebull, L. M., Boesgaard, A. M., Deliyannis, C. P., Hobbs, L. M., King, J. R., \& Ryan, S. G. 1997, ApJ, 488, 338

García López, R. J., Lambert, D. L., Edvardsson, B., Gustafsson, B., Kiselman, D., \& Rebolo, Rafael 1998, ApJ, 500, 241

Kiselman, D. \& Carlsson, M. 1996, A\&A 311, 680

Kohl, J. L., Parkinson, W. H., \& Withbroe, G. L. 1977, ApJ, 212, L101

Lodders, K., Palme, H., \& Gail, H-P. 2009, Landolt-Bornstein, New Series, Astronomy and Astrophysics, Ed. Springer Verlag, in press (arXiv:astro-ph/0901.1149)

Mendel, J. T., Venn, K. A., Proffitt, C. R., Brooks, A. M., \& Lambert, D. L. 2006, ApJ, 640, 1039

Primas, F., Duncan, D. K., Peterson, R. C., \& Thorburn, J. A. 1999, A\&A, 343, 545

Proffitt, C. R. \& Quigley, M. F. 2001, ApJ, 548, 429

Reeves, H., Fowler, W. A., \& Hoyle, F. 1970, Nature, 226, 727

Venn, K. A., Brooks, A. M., Lambert, D. L., Lemke, M., Langer, N., Lennon, D. J., \& Keenan, F. P. 2002, ApJ, 565, 571

Woosley, S. E., Hartmann, D. H., Hoffman, R. D., \& Haxton, W. C. 1990, ApJ, 356, 272 